\title{
Security Issues in Big Data with Cloud Computing
}

\author{
Anitya Kumar Gupta ${ }^{1 *}$, Srishti Gupta ${ }^{2}$ \\ ${ }^{1 *}$ Computer Science Engineering, Apeejay Stya University, Gurugram, India \\ ${ }^{2}$ Electronics and Communication Engineering, Bharti Vidyapeeth College of Engineering, Delhi, India \\ Corresponding Author: Anitya.gupta320@gmail.com, Tel: +91-9915966844
}

Available online at: www.isroset.org

Received: 07/Nov/2017, Revised: 19/Nov/2017, Accepted: 14/Dec/2017, Published: 31/Dec/2017

\begin{abstract}
In this paper, we examine security issues for cloud computing, Big Data, Map Reduce and Hadoop environment. The fundamental focus is on security issues in distributed computing that are connected with huge information. Big data information applications are an awesome advantage to associations, business, organizations and numerous substantial scale and little scale ventures. We additionally talk about different conceivable answers for the issues in distributed computing security and Hadoop. Distributed computing security is creating at a quick pace which incorporates PC security, system security, data security, and information protection. Distributed computing assumes an extremely indispensable part in securing information, applications and the related foundation with the assistance of arrangements, innovations, controls, and enormous information instruments. In addition, distributed computing, enormous information and its applications, focal points are prone to speak to the most promising new frontiers in science.
\end{abstract}

Keywords ----- Big Data, Cloud Computing, Hadoop, HDFS (Hadoop Distributed File System), Map Reduce.

\section{INTORODUCTION}

Keeping in mind the end goal to break down complex information and to distinguish designs it is vital to safely store, oversee and share a lot of complex data. Cloud accompanies an express security challenge, i.e. the information proprietor won't not have any control of where the information is set. The reason behind this control issue is that on the off chance that one needs to get the advantages of distributed computing, he/she should additionally use the assignment of assets furthermore the booking given by the controls. Consequently, it is required to secure the information amidst conniving procedures. Since cloud includes broad multifaceted nature, we trust that as opposed to giving an all-encompassing answer for securing the cloud, it is perfect to make essential upgrades in securing the cloud that will at last furnish us with a safe cloud. Google has presented Map Reduce [1] structure for preparing a lot of information on item equipment. Apache's Hadoop circulated record framework (HDFS) is advancing as an unrivalled programming segment for distributed computing joined alongside coordinated parts, for example, Map Reduce. Hadoop, that's an open-supply usage of Google Map Reduce, together with a dispersed report framework, offers to the utility developer the deliberation of the manual and the lessen. With Hadoop it is less demanding for associations to take a few to get back some composure on the huge volumes of information being produced every day, except in the meantime can likewise make issues identified with security, information access, observing, high accessibility and business coherence. In this paper, we think of some methodologies in giving security. We should a framework that can scale to handle countless furthermore have the capacity to process expansive and enormous sums of information. Be that as it may, cutting edge frameworks using HDFS and Map Reduce are not exactly enough/adequate due to the way that they don't give required efforts to establish safety to ensure delicate information. Besides, Hadoop system is utilized to take care of issues and oversee information helpfully by utilizing diverse systems, for example, consolidating the kimplies with information mining innovation [2].

\section{CLOUD COMPUTING}

It is a revolution which is predicated on share of property which is data than have closed by servers or person is device required to handle the package. In Cloud Computing, the phrase "Cloud" indicates "The internet", so Cloud Computing implies a kind of registering wherein administrations are conveyed via the internet. The objective of Cloud Computing is to make utilization of expanding registering energy to execute a huge number of guidelines every second. Distributed computing utilizes systems of an extensive gathering of servers with specific associations with circulate information handling among the servers. Rather than introducing a product suite for every PC, this innovation 
requires to introduce a solitary programming in every $\mathrm{PC}$ that permits clients to sign into a Web-based administration and which likewise has every one of the projects required by the client. There's a critical workload shift, in a cloud processing framework. Neighbourhood PCs no more need to take the whole weight with regards to running applications. Distributed computing innovation is being utilized to minimize the use expense of figuring assets [4]. The cloud system, comprising of a system of PCs, handles the heap. The expense of programming and equipment on the client end diminishes. The principle element that have to be accomplished on the client's stop is to run the cloud interface programming to partner to the cloud. Disbursed computing contains of a front end and returned give up.

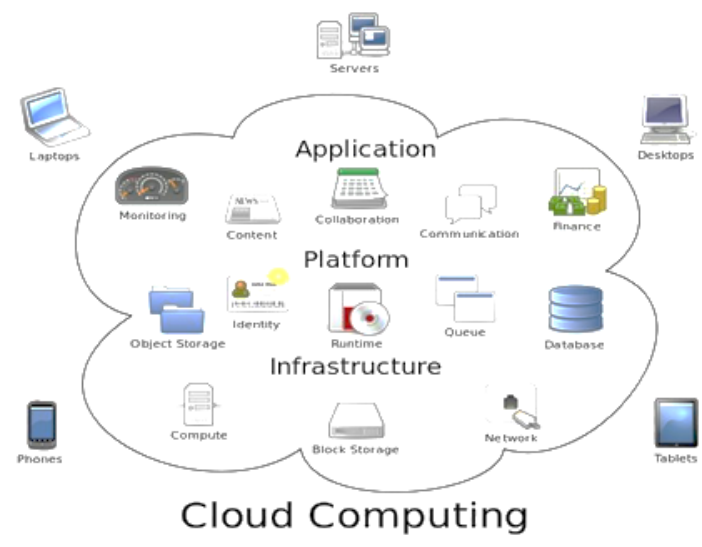

Figure-1. CLOUD COMPUTING

The front end incorporates the user's computer and programming required to get to the cloud system. Back end comprises of different PCs, servers and database frameworks that make the cloud. The purchaser can get to packages within the cloud machine from anyplace by using interfacing with the cloud making use of the internet.

\section{BIG DATA}

Huge Data is the word used to portray enormous volumes of organized and unstructured information that are large to the point that it is exceptionally hard to process this information utilizing conventional databases and programming innovations. The expression "Enormous Data [5]" is organizations who needed to inquiry approximately organized vast circulated information.

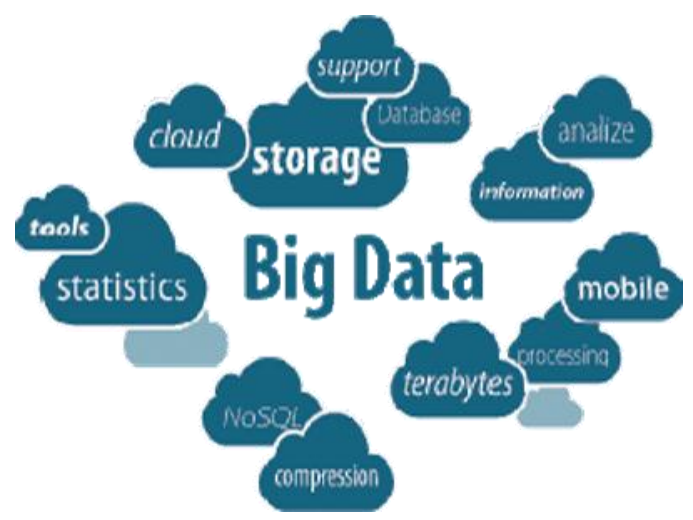

Figure-2. BIG DATA

The three primary terms that connote Big Data have the accompanying properties:

a) Volume: Many components contribute towards expanding Volume spilling information and information gathered from sensors and so forth.

b) Variety: Today information comes in a wide range of organizations messages, video, sound, exchanges and so on.

c) Velocity: This implies how quick the information is being created and how quick the information should be handled to take care of the demand. The other two measurements that need to consider regarding Big Data Are Variability and Multifaceted nature [5].

d) Variability: At the side of the rate, the statistics flows can be fairly inconsistent with periodic peaks...

e) Complexity: Complexity of data had to be taken into observation as data is generated from the unique resources. The information must be connected, coordinated, washed down and changed into required designs before real preparing. Advancements today not just backing the accumulation of a lot of using such information adequately. Exchanges made everywhere throughout the world as for a Bank, Walmart client exchanges, and Facebook clients creating social association information.

\section{HADOOP}

Hadoop, which is a free, Java-based programming structure, underpins the preparing of expansive sets of information in a circulated registering environment. It is a piece of the Apache project supported by the Apache Software Foundation. Hadoop institution utilizes a master/Slave shape [6]. Utilizing Hadoop, extensive information sets can be handled over a bunch of servers and applications can be keep running on frameworks with a great many hubs including thousands offer 
a byte. Disseminated record framework in Hadoop helps in fast information exchange rates and permits the framework to proceed with its ordinary operation indeed, even on account of some hub disappointments. This methodology brings down the danger of a whole framework disappointment, indeed, even on account of a critical number of hub disappointments. Hadoop empowers a registering arrangement that is versatile, practical, a datable and shortcoming tolerant. Hadoop Framework is utilized by well-known organizations like Google, Yahoo, Amazon and IBM and so forth. To bolster their applications including tremendous measures of information. Hadoop has two principle sub ventures - Map Reduce and Hadoop Distributed Record System (HDFS).

\section{MAP REDUCE}

Hadoop Map Reduce is a structure [7] used to compose applications that procedure a lot of information in parallel on groups of ware equipment assets in a dependable, deficiency tolerant way. A Map lessen work first partitions the information into individual portions that are dealt with by means of Map employments in parallel. The yields of the maps sorted by the system are then contribution to the lessen assignments. By and large the info and the yield of the employment are both put away in a document framework. Booking, Observing and re-executing fizzled undertakings are taken consideration by the structure.

\section{HADOOP DISTRIBUTED FILE SYSTEM}

HDFS [8] is a document framework that traverses every one of the hubs in a Hadoop group for information stockpiling. It joins together document frameworks on nearby hubs to make it into one expansive record framework. HDFS moves forward dependability by duplicating information over different sources to conquer hub disappointments. Information applications, a calculated system of prescient assembling starts with information securing where there is a plausibility to get distinctive sorts of tactile information, for example, weight, vibration, acoustics, voltage, current, and controller information. The blend of tactile information and verifiable information builds the enormous information in assembling. This created enormous information from the above blend goes about as the contribution to prescient apparatuses and preventive systems, for example, prognostics what's more, wellbeing administration. The imperative application of it is Bioinformatics which covers the people to come sequencing and other organic spaces. Bioinformatics which requires a vast scale information examination utilizes Hadoop. Distributed computing gets the parallel dispersed figuring structure together with PC groups and web interfaces.

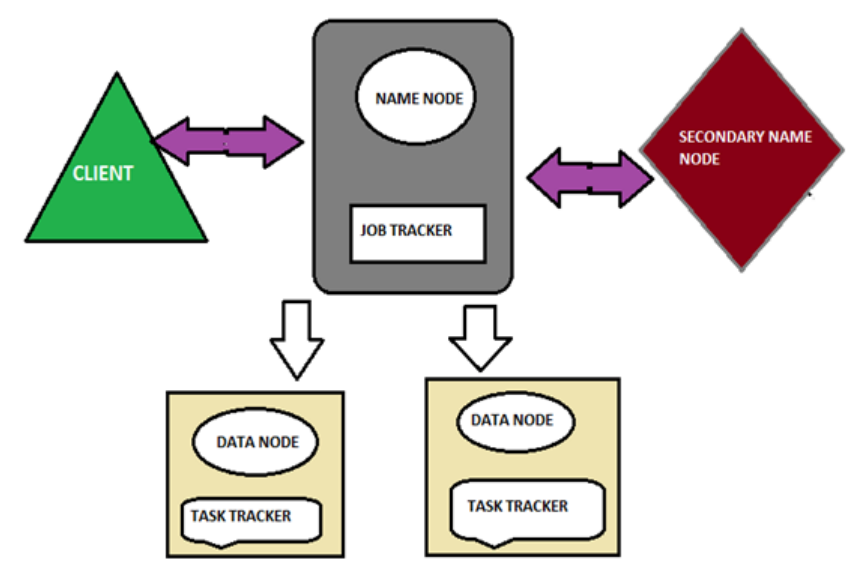

Figure 1 HDFS

\section{BIG DATA ADVANTAGES}

In Big information, the product bundles give a rich arrangement of instruments and alternatives where a person could outline whole information scene over the organization, in this manner permitting the person to break down the dangers he/she confronts inside. This is considered as one of the principle favourable circumstances as large information keeps the information safe. There are some normal attributes of enormous information, for example,

- Big information coordinates both organized and unstructured information.

- Addresses pace and versatility, portability and security, adaptability and steadiness.

- In huge information the acknowledgment time to data is basic to concentrate esteem from different information sources, including cell phones, radio recurrence recognizable proof, the web and a developing rundown of mechanized tangible advancements.

\section{NEED OF SECURITY IN BIG DATA}

In this new period, numerous organizations are utilizing the innovation to store and investigate peta bytes of information about their organization, business and their clients. Thus, data characterization turns out to be much more basic. For making enormous information secure, systems, for example, encryption, logging, honey pot identification must be vital. In numerous associations, the arrangement of enormous information for misrepresentation location is extremely alluring and valuable. The test of recognizing and counteracting propelled dangers and vindictive gate crashers, must be settled utilizing enormous information style examination. These methods help in distinguishing the dangers in the early stages utilizing more complex example investigation and breaking down various information sources. 
Security as well as information protection challenges existing enterprises and government associations.

\section{DEMANDING SITUATIONS}

The technology of Cloud Computing acknowledges different security problems because it comprise of various innovations united with different designs, databases, virtualization and memory management. Consequently, security issues of these frameworks and innovations are relevant to cloud computing. The challenges of security in distributed computing situations can be ordered into system level, client confirmation level, information level, and generic issues.

Network level: The difficulties that can be classified under a system level manage system conventions and system security, for example, dispersed hubs, and circulated information, Internodes correspondence.

Authentication level: The difficulties that may be looked after beneath customer affirmation stage preparations with encryption/unscrambling strategies, confirmation strategies together with administrative rights for hubs, verification of utilizations and hubs, and logging.

Data level: The difficulties that can be ordered under information level manage information honesty and accessibility, for example, information insurance and conveyed information.

Generic types: The difficulties that can be classified under general level are Conventional security instruments and use of various innovations.

\section{PRAPOSED APPROACHES}

Following security measures should be taken to ensure the security in a cloud environment.

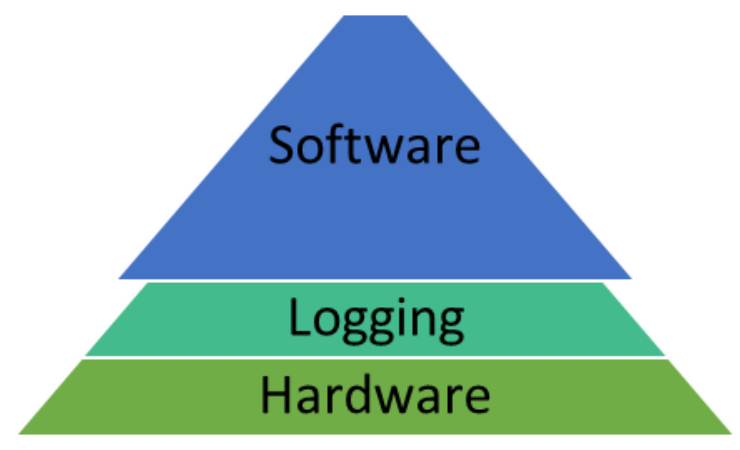

Figure 2THREE LAYER SECURITY ARCHITECTURE

\section{A. Software:}

File Encryption: Since the information is available in the machines in a bunch, a programmer can take all the basic data. In this manner, every one of the information put away ought to be scrambled. Various encryption keys have to be applied on diverse machines and the key information have to be put away midway in the back of stable firewalls. Along these lines, regardless of the possibility that a programmer can get the information, he can't extricate significant data from it and abuse it. Client information will be put away safely in an encoded way.

Network Encryption:- All the system correspondence ought to be encoded according to industry gauges. The RPC method calls which occur ought to happen over SSL so that regardless of the possibility that a programmer can take advantage of system correspondence bundles, he can't extricate helpful data or control parcels.

Software Format and Node Maintenance: Hubs which run the product ought to be designed frequently to dispose of any infection present. All the application programming projects and Hadoop programming ought to be redesigned to make the framework more secure.

Rigorous System Testing of Map Reduce Jobs: After a clothier composes a manual diminish paintings, it need to be absolutely attempted in a dispersed environment as opposed to a solitary gadget to guarantee the energy and soundness of the profession.

Honey pot Nodes: Nectar pot hubs ought to be available in the group, which seem like a normal hub yet is a trap. These honey pots trap the programmers and essential moves would be made to take out programmers.

\section{B. Logging:}

Log:-All the guide lessens occupations which alter the information ought to be logged. Likewise, the data of clients, which are in charge of those occupations, ought to be logged. These logs ought to be evaluated routinely to find assuming any, noxious operations are performed or any vindictive client is controlling the information in the hubs.

Nodes Authentication: At whatever point a hub joins a group, it ought to be confirmed. If there should be an occurrence of a noxious hub, it ought to not be permitted to join the group. Confirmation procedures like Kerberos can be utilized to approve the approved hubs from vindictive ones

Layered Framework for Assuring Cloud: A layered structure for guaranteeing distributed computing comprises of the 
secure virtual machine layer, secure distributed storage layer, secure cloud information layer, and the safe virtual system screen layer. Cross cutting administrations are rendered by the strategy layer, the cloud observing layer, the unwavering quality layer and the danger investigation layer.

Publication of security in context of third party: Dispensed computing helps in setting away of data at a far flung website online in order to expand asset utilization. Accordingly, it is imperative for this information to be secured and get to ought to be offered just too approved people. Consequently, this in a general sense adds up to secure outsider distribution of information that is required for information outsourcing, and also for outside productions

Access Control:- Reconciliation of compulsory access control and differential security in circulated environment will be a decent security measure. Information suppliers will control the security strategy of their delicate information. They will likewise control the scientific bound on security infringement that could occur. In the above methodology, clients can perform information calculation with no spillage of information. To forestall data spill, SELinux will be utilized. SELinux is only Security-Enhanced Linux, which is a component that gives the instrument to supporting access control security arrangement using Linux Security Modules (LSM) in the Linux Kernel.

\section{Hardware:}

Device Identity:Device Identity plays the significant role when it comes to cyber security. If any individuals phone has been hacked than it can be detected with the help of DI number. Every person gets unique DI which stores the copy of entire data warehouse of the individual and then can be inferred when the cyber-attack is erupted. DI works as tracker which can save data when the hackers approached to retrieve information than artificial identity number which gives you in efficient data.

Secure network scale: Development of Transparent load balancing network devices when the set of data and information categorised in different clusters are been ready for the deployment. Some of the frequent devices been developed are: NG - Firewall, and Intrusion Prevention System. When the performance of the device is not enough than we can add a new bypass called as Inter Traffic Domain which benefit the flow of the data and ROI of deposition of deployment.

Physical Security:The most of the physical aspect security is handled by the different organisational centres and one of the most popular centre is Operational Intelligence Centre by
Qognify which handles large volumes of data and detect the deviations precursors and events. Sensors are also helpful with BCI protocols which handle the integrated system enterprise with deemed relevant.

\section{CONCLUSION}

Cloud environment is generally utilized as a part of industry and examination viewpoints; in this manner security is a vital viewpoint for associations running on these cloud situations. Utilizing proposed approaches, cloud situations can be secured for complex business operations. We can also examine that when it comes to hardware security than advance featured technology like machine learning, brain astronomical BCI protocols and different advance programming languages are used to analyse data warehouse which makes the time complexity in nano scales. The upcoming technological world will be fully concentrated on the priorities on security and the managing about issues related to security and configuration management pertaining to the conflict of handling big data will be given great fight in producing the new versions of securities.

\section{REFERENCES}

[1] Ren, Yulong, and Wen Tang. "A Service Integrity Assurance Framework For Cloud Computing Based On Mapreduce."Proceedings of IEEE CCIS2012. Hangzhou: 2012, pp 240 -244, Oct. 30 2012-Nov. 12012

[2]N, Gonzalez, Miers C, Redigolo F, Carvalho T, Simplicio M, de Sousa G.T, and Pourzandi M. "A Quantitative Analysis of Current Security Concerns and Solutions for Cloud Computing.". Athens: 2011. pp 231 - 238, Nov. 29 2011- Dec. 12011

[3] A. Haripriya, A.P. Siva Kumar, "An Effective Patient Treatment Plan Recommendation with Predicted Treatment Time Using Hadoop", International Journal of Computer Sciences and Engineering, Vol.5, Issue.8, pp.155-158, 2017.

[4] Ujjwal Agarwal, "Cloud Computing: BDaaS and HDaaS (Big Data as a Service and Hadoop as a Service)", International Journal of Computer Sciences and Engineering, Vol.5, Issue.11, pp.131-134, 2017.

[5] "Security-Enhanced Linux." Security-Enhanced Linux. N.p. Web. 13 Dec 2013.

[6] Big data adoption- Infrastructure consideration -TCS White paper

\section{AUTHORS PROFILE}

CFE - IFS Anitya Gupta pursuing Bachelor of Technology in Cloud Computing from Apeejay Stya University. He is currently working as research fellow in Defence Research and Developemnt Organisation - Defence Body of India. His key research field is Artificial Intelligence. His expertise is in Virtual Reality, Quantum Programming, Brain Astronomy. Securities and Designing Algorithms. He has publish more than 10 research papers in reputed international journals 
acroos world and also in IEEE. He is a life member of ISROSET since 2017 and also the member of INBA in field of forensics. He has been granted the Certified Forensic Expert from International Forensic Scieneces and Scrum Product Owner from Scrum Institute Switzerland.

Ms. Srishti Gupta is the PAN India Student Manager for Internshala and is pursuing her Bachelor's degree in Electronics and Communication Engineering from Bharati Vidyapeeth's College of Engineering, Delhi under IP University. She is also the Vice-President of Association for Computing Machinery- Women's Council, BVP and Microsoft's Student Partner. She has also worked as Project Leader for UNFCCC Green Revolution Program with ICCE and heads the National Service Scheme in her college, besides being the TCS Campus Ambassador. Having completed 13 internships in 2.5 years alongside full time studies, she has also been the Panelist for National Start-up pitch held at NASSCOM and IAMAI, Bangalore. 